\title{
KINETIC SOLUBILITY OF FISETIN NANOCRYSTAL
}

\author{
Muhammad Dzakwan', Ganet Eko Pramukantoro', Rachmat Mauludin², Saleh Wikarsa² \\ ${ }^{1}$ Faculty of Pharmacy, Universitas Setia Budi, Surakarta, Central Java, Indonesia \\ ${ }^{2}$ Department of Pharmaceutics, School of Pharmacy, Institute Technology Bandung \\ Labtek VII Ganesha 10 Bandung West Java, Indonesia \\ Corresponding author: mdzakwan9@setiabudi.ac.id
}

\begin{abstract}
Background: Fisetin (3,3,4,7-tetrahydroxyflavone) is a natural antioxidanthaspoor solubility leads to poorbioavailability and limits its development. Fisetinnanocrystals were produced by nanoprecipitation technique and transformed into dry powder by lyophilisation. Objective: The aim of the study is to investigate the kinetic solubility of fisetinafter produced into nanocrystals. Methods: Fisetin nanocrystalswere prepared by nanoprecipitationwith different proportion of stabilizers and transformed into dry powder by lyophilization. In a period of 1 week, the kinetic solubility was determined using a shaker water bath at $37^{\circ} \mathrm{C}$. Result: Showed fisetin nanocrystals were almost completely dissolved within $15 \mathrm{~min}$ in water and buffer $\mathrm{pH} 1,2$. In contras, only $55 \%$ of fisetin raw material at the same condition. Conlusions: Lyophilizedfisetinnanocrystals could be re-dispersed completely in water, buffer $\mathrm{pH} 1,2$ and the kinetic solubility in water increased to $420 \mathrm{ng} / \mathrm{ml}$.
\end{abstract}

\section{Keywords: fisetin, nanocrystal, nanoprecipitation, kinetic solubility}

\section{Introduction}

Flavonoids, e.g., fisetin, luteolin and kaemferol comprise a vast group of naturally occurring polyphenolic compounds, considered as active pharmaceutical ingredients (APIs), and present in daily consumed vegetables, fruits and products of that origin (Scholz and Keck, 2014., Chen et all,2014). Fisetin (FIS, 3,7,3',4'tetrahydroxyflavone, Fig 1) belongs to the flavonol subgroup of flavonoids found in 65 many vegetables and fruit and is especially rich in apples, strawberries, onions and mangoes (Seguin et all, 2013., Wang et all., 2016). It exhibits antioxidant, anti-inflammatory and anticarcinogenic activities. Recently, it was also considered to possess neuroprotective effects against the aging process, cerebral damage and neurodegenerative disorders (Ragelle et all, 2012., Wang et all, 2016). However, a limiting factorfisetin ispoor solubility, restricting their full antioxidative potential as well as bioavailability (Scholz and Keck, 2014). The drug has dissolution rate limited bioavailability and therefore formulating as nanosuspension might improve its dissolution rate thereby enhance oral bioavailability (Kumar et al, 2008).<smiles>O=c1c(O)c(-c2ccc(O)c(O)c2)oc2cc([Te])ccc12</smiles>

Figure 1-Chemical structure of fisetin (fisetin(Ragelle et all, 2012)

Over the last decade, there has been increase in thenumber of newly developed drug molecules that exhibit poor water solubility as well as poor bioavailability. So, one of the most challenging tasks indrug development is to improve solubility and oral bioavailability of these novel drugs. Various methods to enhance solubility of poorly water-soluble drugs include solubilization (Dulfer et al,1995), formation of complexes $\beta$ cyclodextrin (Calabro et al, 2005), solid dispersions (Kanaze et al, 2006), liposomes (Dupont,2002), emulsions (Nakano, 2000)and microemulsions (Lawrence and Rees, 2000). However, these methods are successful in some instances and are specific to drug candidates. 
The fundamental basis for the observation that size influencesthe solubility is found in the Kelvin equation (Buckton and Beezer, 1992). The Kelvin equation is equally applicable to the solid-liquid interface, because the vapor pressure in a liquid-gas system corresponds to the dissolution pressure in a solid-liquid phase system. Replacing the pressure terms with solubilities (cs), as activity coefficients are effectively set equal to the unity in the dilute solutions considered here, produces the so-called Gibbs-Kelvin relation.

The aim of this studyis to assess of kinetic saturation solubilityoffisetinnanocrystal.The physicochemical properties of thefisetinnanocrystals and the nanocrystal powder have been investigated regarding crystallinity and kinetic solubility.

\section{Methods}

\section{Materials}

Fisetin (98\%) raw material was purchased from Shaanxi Dideu Medichem Co. Ltd (Xi'an, China). Eudragit was obtained from BASF (German). (Sodium lauryl sulphate (SLS) was obtained from Cognis Indonesia Ltd.Polysorbate 80 (Tween $80 \AA$ ) was obtained from Shino Japan Chemical. All other reagents were of analytical grade.

Methods

\section{Production of fisetin nanosuspensions}

FIS were prepared by a precipitation method with different proportion of stabilizers fixed. The suspensions were prepared by dissolving $0.01 \mathrm{~g}$ of FIS in $2.0 \mathrm{~mL}$ of ethanol as the organic phase. Then the organic phase was injected slowly at a stirring rate of $600 \mathrm{rpm}$ using a magnetic stirrer (RCT Basic, IKA, Staufen, Germany) into $20 \mathrm{~mL}$ of aqueous phase containing polysorbat 80 and SLS (Table I).

\section{Particle size analyses}

The particle size and zeta potential value of the mebendazole nanosuspensions were determined by a Zetasizer Nano ZS (Malvern Instrument $\mathrm{UK}$ ).The samples were adequately diluted with deionised water and placed in an electrophoretic cell.

\section{Lyophilization}

Thefisetinnanosuspensions were dried by lyophilization. Eachfisetinnanosuspension (10 $\mathrm{mL}$ ) was freeze-dried at $-80^{\circ} \mathrm{C}$ in a $20 \mathrm{~mL}$ vial using Martin Christ " 1-2/LD plus freeze dry system (Martin Christ Gefriertrocknungsanlagen GmbH, 37507 Osterodeam Harz, Germany) with 50 mbar vacuum for $24 \mathrm{~h}$. The dried powder was stored in a desiccator.

\section{Characterization by DSC}

Differential scanning calorimetric (DSC) measurements were carried out using a TA DSC 60 thermal analyzer (TA-60 WS DE 19720, Shimadzu, JAPAN) in a temperature range of $30^{\circ} \mathrm{C}-300^{\circ} \mathrm{C}$ at a heating rate of $10^{\circ} \mathrm{C} / \mathrm{min}$ in nitrogen gas

\section{Powder X-ray diffraction (PXRD)}

$\mathrm{X}$-ray diffraction was studied using the Bruker AXS D8 Advance X-ray diffractometer (Bruker AXS, Madison, USA) $\mathrm{Cu} \mathrm{K}^{\prime \prime}$ targets at a scanning rate of $0.0102 \theta / \mathrm{s}$, applying $40 \mathrm{kV}$ at 40 $\mathrm{mA}$, to observe the crystallinity of samples.

\section{Kinetic solubility}

Solubility studies were performed with shaking water bath (SW 22 Shaking Water Bath, Julabo GmbH, Eisenbahnstr, Germany ) at 370 C. Vials were sealed to avoid changes due to evaporation and placed for 1 week in a thermostated storage at $37 \pm 0,5^{\circ} \mathrm{C}$. After the equilibrium was reached, suspensions were filtered through Sartorius ${ }^{\circledR} \quad 0.1 \mathrm{~mm}$ filters (Sartorius AG, Germany). An aliquot from each vial was withdrawn by a $1 \mathrm{ml}$ glass syringe (Poulten \& Graf GmbHGermany) and assayed by spectrometrically at $287 \mathrm{~nm}$ using an ultraviolet spectrometer (Beckman D270, Beckman Coulter GmbH, Germany) to evaluate the amount of FIS dissolved.

Table 1. Composition of FIS nanosuspension

\begin{tabular}{llcc}
\hline & \multicolumn{3}{l}{ Formulation (\%) } \\
\cline { 2 - 4 } & A & BC \\
\hline FIS & 1 & 1 & 1 \\
SLS & 0.5 & - & - \\
Tween 80 & - & 0,5 & - \\
Euderagit & - & - & 1 \\
Water & 98.5 & 98.5 & 98 \\
\hline Result And Discussion
\end{tabular}

\section{Result And Discussion}

Production of aqueous nanosuspensions and Lyophilization

FIS nanosuspensions stabilized by tree different stabilizers were successfully produced. The PCS particle size result of allnanosuspensions are shown in Table 2.The nanosuspension stabilized by SLS (formulation A) was chosen for further investigations. Dried 
FIS nanocrystals were obtained bylyophilization using the freeze dryer. After $24 \mathrm{~h}$, lyophilized FIS nanocrystals were collected. The physical characteristics of the lyophilized FIS nanocrystals were investigated with regard to crystalline state and kinetic solubility.

Table2. Mean PCS particle size and PI FIS nanosuspension

\begin{tabular}{llll}
\hline & \multicolumn{3}{l}{ Formulation } \\
\cline { 2 - 4 } & A & B & C \\
\hline z-average (nm) & 402.8 & 225.7 & 1019.1 \\
PI & 0,212 & 0,253 & 0,277
\end{tabular}

\section{Crystalline state evaluation by DSC}

Thermal analysis of the FIS nanocrystal powder was performedand compared to the raw material and to the data in the literature.The FIS nanocrystal powders investigatedwere obtained by lyophilization. The results of thermal analysis by DSC can be seen in Fig. 2.According to these results, the melting points of FIS (raw material and both nanocrystal powders) are similar. The only difference observedwas a slight shift in melting temperature (from $320,53^{\circ} \mathrm{C}$ to $323^{\circ} \mathrm{C}$ ). This modification was attributed to the presence of SLS as stabilizer and mannitol as cryoprotectant. The results of the thermal analyses are shown the melting points of the FIS nanocrystals have not changed significantly. The presence of stabilizers has a minor influence on the melting temperature and has not altered the crystallinity of the drug

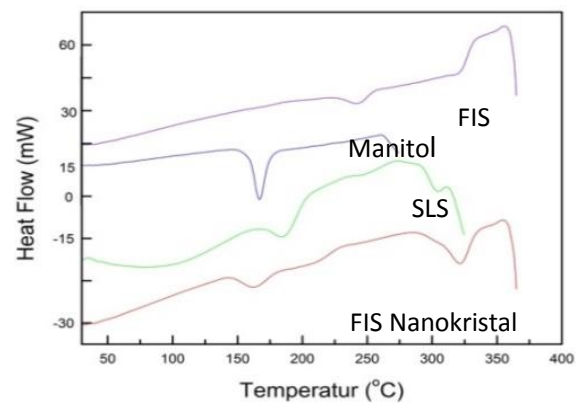

Figure 2 - DSC thermogram of lyophilized, FIS nanocrystals and raw material

\section{Crystalline state evaluation by XRD}

To confirm the crystalline state of the dried FIS nanocrystals, X-ray diffractionwas performed of the lyophilized FIS nanocrystals. X-ray diffraction patterns were visualized in diffractograms can be seen in Fig. 3. The crystallinity of lyophilized FIS nanocrystals with SLS as stabilizers. The diffractograms reveal no different peaks for FISnanocrystals. According to these results, FIS nanocrystals were still in the same crystalline state as the raw material. Applying energy by being homogenized and subjecting them to the drying processes did not transform FIS nanocrystals to be fully or partially amorphous. In general, more crystalline substances are physically more stable compared to amorphous forms.

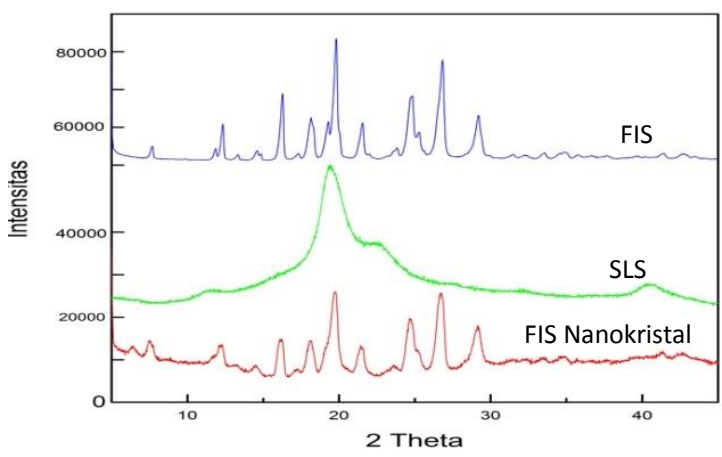

Figure 3 - X-ray diffractogram patterns of lyophilizedFIS nanocrystal formulations and the raw material

\section{Kinetic solubility}

Thermodynamic solubility is the concentration in thesolute in equilibrium with a normal sized powder, this condition being physically stable. Kinetic solubility, e.g. achieved by size reduction or from amorphous powders, is higher than the thermodynamic solubility but physically not long-termstable (Mauludin et al, 2009).The kinetic solubility of FISnanocrystals was investigated over 1 week. The results of this testing showed that the solubility of the FIS nanocrystals in water and $\mathrm{pH} 1.2$ were increased at $370 \mathrm{C}$. The kinetic solubility was with $420 \mu \mathrm{g} / \mathrm{ml}$ higher than of the raw material $(60,57 \mu \mathrm{g} / \mathrm{ml})$ (Fig. 4).

According to the crystalline state investigation, the solubility enhancement of the FIS nanocrystalsis not due to the presence of the FIS amorphous form. It is due to the particle size reduction to the submicron range. This result is in agreement with the Kelvin-Gibbs equation and Ostwald-Freundlich equation 
(Buckton and Beezer, 1992; Müller and Akkar, 2004)

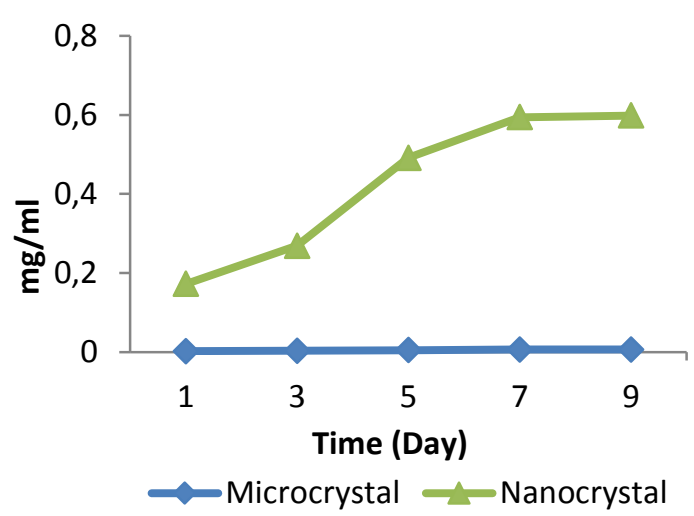

Figure 4 - Kinetic saturation solubility of the FISnanocrystalsand FIS microcrystals in water at $37^{\circ} \mathrm{C}$.

\section{Conclusion}

FIS nanocrystals could provide superior physicochemicalproperties.Lyophilized FISnanocrystals improved the kinetic saturation solubilityfrom $60,57 \eta \mathrm{g} / \mathrm{ml}$ to $420 \eta \mathrm{g} / \mathrm{ml}$, comparison to FIS microcrystals.

\section{References}

Buckton G \& Beezer A E., 1992, Int. J. Pharm, The relationship between particle size and solubility, 82, R7-R10.

CalabroML. et al., 2005,J Pharm Biomed Anal, The rutin/beta-cyclodextrin interactions in fully aqueous solution: Spectroscopic studies and biological assays, 36:1019-1027.

Chen P.Y, Ho Y.R, Wu M.J, Huang S.P, Chen P.K, Tai M.H, Ho C.T and Yen J. H 2014, Cytoprotective Effects of Fisetin Against Hypoxia-Induced Cell Death in PC12 Cells, Food\& Function 1:1-10.

DulferWJ. et al., 1995, Environ Sci Technol, Micellar solubilityand micelle/water partitioning of polychlorinated biphenyls in solutions of sodium dodecyl sulphate, 29:985-992.

Dupont B., 2002,J Antimicrob Chemother Suppl, Overview of the lipid formulations of amphotericin B. S1:31-36.

Eskandari M. et al., 2011, Journal of Pharmaceutical and Biomedical Analysis,Microextraction of mebendazole across supported liquid membrane forced by pHgradient and electrical field, 1173-1179

Kanaze FI. et al., 2006, J Appl Polym Sci, Dissolution enhancement of flavonoids by solid dispersion in PVP and PEG matrixes: A comparative study, 102:460-471.

Kumar M.P. et al., 2008, Current NanoscienceFormulation of Nanosuspensions of Albendazole for Oral Administration,4, 53-58

Lawrence M \&Rees G., 2000, Adv Drug Deliv $\mathrm{R} e v$, Microemulsion-based media as novel drug delivery systems, 45:89-121.

Mauludin R et al., 2009, Eur J of Pharm Sci, Kinetic solubility and dissolution velocity of rutin nanocrystals, $36: 502-510$

Müller R H \& Akkar A., 2004. Drug nanocrystals of poorly solubledrugs. In: Nalwa, H.S. (Ed.), Encyclopedia of Nanoscience and Nanotechnology. American Scientific Publishers, 627-638.

Kumar M.P. et al., 2008, Current NanoscienceFormulation ofNanosuspensions of Albendazole for Oral Administration,4, 53-58

Nakano M., 2000, Adv Drug Deliv Rev, Places of emulsions in drug delivery, 45:1-4.

RagelleH,Manciet S.C, Seguin J, Brossard D, Scherman D, Arnaud P, Guy G and ChabotG.G2012, Nanoemulsion Formulation of Fisetin Improves Bioavailability and Antitumor Activity in Mice, International Journal of Pharmaceutics, 427:452- 459

Scholz Pand Keck C.M2014, Flavonoid Nanocrystal Produced byARTcrystal ${ }^{\circledR}$ Technology, International Journal of Pharmaceutics1447:1-11

Seguin J, Brullé L, Boyer R, Lu Y.M, Romano M.R, Touil Y.S, Scherman D,Bessodes M,Mignet $\mathrm{N}$ and Chabot G. G2013, Liposomal Encapsulation Of The Natural Flavonoid Fisetin Improves Bioavaibility and Antitumor Efficacy,International Journal of Pharmaceutics444:146-154

Wang L, Zhang D.Z and Wang Y.X2016, Bioflavonoid Fisetin Loaded $\alpha$-TocopherolPoly(lactic acid)-Based Polymeric Micelles for Enhanced Anticancer Efficacy in Breast Cancers, Pham Re 